\title{
GENETIC VARIABILITY AND PATH COEFFICIENT IN MAIZE (Zea mays L.) GENOTYPES
}

\author{
Hafiz Saad Bin Mustafa', Muhammad Aslam² ${ }^{2}$ Ejaz-ul-Hasan', Fida Hussain ${ }^{1}$ \\ and Jehanzeb Farooq ${ }^{3}$
}

\begin{abstract}
Present investigations were aimed at estimating the genetic parameters and character's association in forty maize genotypes. The PCV was higher in magnitude than GCV for almost all the traits except for root density which showed greater value of GCV than PCV. Heritability estimates were also higher in all the traits and highest hretability was shown for chlorophyll content. Path coefficient analysis revealed that the fresh shoot length had maximum direct effect on fresh root length followed by root density, dry shoot weight, leaf temperature and dry root weight. It may be concluded that fresh root length, dry shoot weight, root density, leaf temperature and dry root weight are the characters which contribute largely to the fresh shoot length of maize seedlings. These traits had reasonable heritability estimates, thus selection could be made for high yielding maize genotypes on the basis of these traits.
\end{abstract}

Key words: Zea mays, path coefficient, heritability, genetic advance, direct effect

\section{INTRODUCTION}

Maize is an important cereal crop and ranks third among cereal crops after wheat and rice. In Pakistan, maize is grown on an area of 1083 thousand hectares with total production of 3990 thousand tons (Anonymous, 2011-12). It is consumed as food by human and feed for the livestock and poultry. It also fulfills the requirement of raw material in food, medicine and textile industries, which finally manufacture corn oil, corn flakes, dextrose, textile dyes etc. Maize has high nutritive value as it contains $72 \%$ starch, $10 \%$ protein, 4.80 $\%$ oil, $8.50 \%$ fiber, $3.0 \%$ sugar, and $1.70 \%$ ash (Chaudhary, 1983). Selection is the oldest method and its success in plant breeding largely depends upon many factors such as the interrelationship of various traits (Ilker, 2011).

The exploitation of maize inbreds for genetic analyses requires a detailed knowledge of genetic and historical relationships between these lines and an understanding of the partitioning of genetic diversity among them. For example, developmental mutants of maize can exhibit strikingly different phenotypes when assayed in the genetic backgrounds of different maize inbred lines (Poethig, 1988). Knowledge of the relationships among lines would help to identify a set of inbreds that have maximal diversity for the analysis of the effects of genetic background. Use of maize inbreds in association analyses requires that population structure among lines be factored into the analysis (Thornsberry et al., 2001).

The present study was planned to estimate the genetic variability, correlation and path coefficient analysis for various characters of maize to select the best genotypes at seedling stage. 


\section{MATERIALS AND METHODS}

The study was carried out in the glass-house of the Department of PlantBreeding and Genetics, University of Agriculture, Faisalabad, Pakistan during 2010. The experimental material was consisting of 40 accessions namely: EV-1098, EV-5098, EV-6098, SWL-2002, Agaiti-2002, Sadaf, Pak Afgoyee, TLOZA, TLOOB-341, TLOOB-343, F-153, F-204, F-220, F-115, F-186, F-135, F-202, F-206, F-209, F-211, F-210, F-192, F-114, B-54, AF-02B, F-109, F-110, F-163, F-208, F-189, F-219, F-158, F-160，F-191，PR-98，8288，8441，6525, $32 \mathrm{~B} 33$ and $33 \mathrm{H} 25$. These accessions were sown in polythene bags $(18 \times 9 \mathrm{~cm})$ filled with sandy loam soil (pH 7.8 and EC 1.7 dS m-1) using Completely Randomized Design with three replications. Field capacity of the soil was determined before sowing. Two seeds per polythene bag were sown and thinned up one healthy seedling after emergence. All the recommended agronomic and cultural practices were carried out. This moisture level was maintained by volume on alternate days by using a moisture meter $(\Delta \mathrm{T}-\mathrm{NH} 2$, Cambridge, England). The average temperature throughout the experimental period was $40{ }^{\circ} \mathrm{C}$. At five leaf stage, five plants were selected randomly from each genotype and data were recorded for the leaf temperature, fresh root length, fresh shoot length, root-to-shoot length ratio, dry root weight, dry shoot weight, root-to-shoot weight ratio, root density and chlorophyll contents and analyzed statistically using analysis of variance technique (Steel et al., 1997). Genotypic and phenotypic correlations were calculated to observe the association between different traits (Kwon and Torrie, 1964). Path coefficient analysis was performed (Dewey and $\mathrm{Lu}, 1959$ ) to assess the association between traits.

\section{RESULTS AND DISCUSSION}

Table 01 showed the mean values of different traits of 40 maize genotypes. The genetic parameters presented in Table 02 indicated that the higher genotypic variance was found for the fresh shoot length (81.38) and lowest for root shoot length ratio (0.01). The highest values of phenotypic variance were obtained for trait fresh shoot length (87.88) and lowest for root shoot weight ratio (0.05). The highest genotypic coefficients of variability were obtained for root density $(244.87 \%)$ followed by chlorophyll content $(130.88 \%)$ while the lowest for leaf temperature $(3.10 \%)$. The highest value of the PCV was found for chlorophyll content (131.08) followed by root density (50.09\%) while lowest value was obtained for the leaf temperature (3.84\%). In the current studies except for leaf tempertaure all the traits showed greater values of $\mathrm{PCV} \%$. So leaf temperature may be used as a selection criterion at seedling stage. Similar results were obtained by Ojo et al. (2006); Moulin, et al., (2009) and Ali et al. (2012). The highest heritability values were found for chlorophyll content $(99.70 \%)$, fresh shoot length $(92.60 \%)$, fresh root length $(83.40 \%)$ and for root density $(80.30 \%)$ while the root shoot weight ratio showed the lowest heritability value (16.20\%). The higher values of heritability of aforementioned traits are indicative that selection can be made on the basis of these traits (Ojo et al. 2006; Moulin et al., 2009 and Ali et al., 2012). 
Table: 01 Mean values of different traitsof maize genotypes.

\begin{tabular}{|c|c|c|c|c|c|c|c|c|c|}
\hline Genotypes & LT & Chl. C & FSL & $\mathrm{RD}$ & FRL & RSR & DSW & DRW & RSWR \\
\hline 1 & 30.80 & 0.03 & 53.33 & 3.00 & 31.67 & 0.59 & 0.63 & 0.57 & 0.92 \\
\hline 2 & 32.87 & 0.02 & 52.67 & 1.67 & 27.33 & 0.52 & 1.00 & 0.90 & 0.91 \\
\hline 3 & 31.57 & 1.04 & 57.67 & 3.00 & 24.33 & 0.42 & 0.77 & 0.60 & 0.78 \\
\hline 4 & 30.77 & 0.03 & 67.00 & 3.33 & 25.33 & 0.38 & 1.00 & 1.17 & 1.19 \\
\hline 5 & 31.13 & 0.04 & 66.67 & 3.00 & 24.67 & 0.37 & 1.07 & 0.83 & 0.78 \\
\hline 6 & 33.67 & 0.03 & 49.33 & 2.00 & 22.33 & 0.45 & 0.77 & 0.77 & 0.98 \\
\hline 7 & 31.53 & 0.08 & 51.00 & 2.67 & 24.00 & 0.47 & 0.73 & 0.60 & 0.84 \\
\hline 8 & 28.50 & 1.06 & 56.33 & 6.67 & 23.33 & 0.41 & 0.77 & 0.73 & 0.96 \\
\hline 9 & 31.13 & 0.08 & 57.33 & 3.00 & 21.67 & 0.38 & 0.67 & 0.63 & 0.95 \\
\hline 10 & 30.83 & 0.13 & 62.33 & 2.67 & 25.00 & 0.40 & 0.80 & 0.66 & 0.84 \\
\hline 11 & 31.50 & 0.04 & 63.33 & 2.33 & 23.67 & 0.37 & 0.80 & 0.77 & 0.96 \\
\hline 12 & 31.80 & 0.03 & 50.67 & 2.33 & 21.00 & 0.41 & 0.67 & 0.57 & 0.85 \\
\hline 13 & 31.00 & 0.08 & 69.00 & 3.00 & 24.00 & 0.35 & 0.87 & 0.67 & 0.81 \\
\hline 14 & 29.33 & 1.04 & 55.33 & 6.33 & 19.00 & 0.34 & 0.70 & 0.60 & 0.86 \\
\hline 15 & 30.80 & 0.07 & 56.00 & 3.00 & 24.33 & 0.43 & 0.77 & 0.76 & 1.00 \\
\hline 16 & 29.33 & 1.06 & 48.67 & 7.00 & 26.00 & 0.53 & 0.70 & 0.53 & 0.79 \\
\hline 17 & 31.73 & 0.02 & 46.33 & 2.67 & 17.67 & 0.44 & 0.90 & 1.07 & 1.28 \\
\hline 18 & 29.77 & 1.08 & 38.00 & 7.33 & 20.33 & 0.53 & 0.63 & 1.47 & 0.72 \\
\hline 19 & 33.23 & 0.03 & 41.67 & 1.67 & 27.67 & 0.66 & 0.43 & 0.37 & 0.88 \\
\hline 20 & 31.43 & 0.06 & 43.67 & 3.00 & 13.00 & 0.30 & 0.73 & 0.50 & 0.69 \\
\hline 21 & 30.77 & 0.02 & 51.67 & 3.00 & 17.33 & 0.33 & 0.67 & 0.50 & 0.75 \\
\hline 22 & 30.00 & 1.03 & 55.00 & 6.33 & 17.00 & 0.31 & 0.77 & 0.90 & 1.18 \\
\hline 23 & 30.60 & 0.16 & 56.00 & 3.33 & 17.67 & 0.31 & 0.77 & 0.70 & 0.92 \\
\hline 24 & 32.50 & 0.17 & 46.67 & 2.00 & 15.67 & 0.33 & 0.63 & 0.50 & 0.82 \\
\hline 25 & 30.43 & 0.08 & 49.33 & 4.00 & 18.00 & 0.36 & 0.77 & 0.60 & 0.80 \\
\hline 26 & 30.77 & 0.08 & 36.33 & 4.00 & 15.33 & 0.42 & 0.60 & 0.40 & 0.69 \\
\hline 27 & 31.23 & 0.24 & 34.33 & 3.67 & 14.67 & 0.42 & 0.60 & 0.67 & 1.29 \\
\hline 28 & 30.70 & 0.13 & 36.33 & 4.00 & 16.00 & 0.44 & 0.47 & 0.47 & 1.01 \\
\hline 29 & 31.37 & 0.12 & 44.33 & 3.33 & 17.67 & 0.40 & 0.60 & 0.50 & 0.83 \\
\hline 30 & 31.77 & 0.07 & 39.67 & 4.00 & 20.33 & 0.52 & 0.63 & 0.47 & 0.77 \\
\hline 31 & 31.17 & 0.04 & 45.33 & 3.00 & 17.00 & 0.38 & 0.70 & 0.50 & 0.72 \\
\hline 32 & 31.60 & 0.06 & 55.67 & 4.00 & 20.33 & 0.37 & 0.70 & 0.63 & 0.91 \\
\hline 33 & 31.30 & 0.06 & 54.67 & 5.00 & 20.67 & 0.38 & 0.67 & 0.60 & 0.92 \\
\hline 34 & 29.03 & 1.12 & 57.33 & 7.67 & 22.67 & 0.40 & 0.77 & 0.63 & 0.84 \\
\hline 35 & 33.10 & 0.04 & 53.33 & 2.67 & 12.67 & 0.24 & 0.53 & 0.53 & 0.85 \\
\hline 36 & 31.43 & 1.09 & 56.00 & 8.00 & 21.33 & 0.38 & 0.90 & 0.63 & 0.83 \\
\hline 37 & 31.50 & 1.12 & 59.00 & 7.67 & 21.33 & 0.36 & 0.70 & 0.57 & 0.82 \\
\hline 38 & 31.37 & 1.11 & 56.00 & 7.33 & 20.33 & 0.36 & 0.63 & 0.53 & 0.90 \\
\hline 39 & 31.07 & 1.02 & 51.67 & 6.33 & 15.00 & 0.29 & 0.57 & 0.43 & 0.81 \\
\hline 40 & 31.33 & 0.04 & 31.33 & 3.67 & 12.33 & 0.39 & 0.23 & 0.20 & 0.89 \\
\hline
\end{tabular}

$\mathrm{LT}=$ Leaf temperature, Chl.C $=$ Chlorophyll contents, $\mathrm{FSL}=$ Fresh shoot length, $\mathrm{RD}=$ Root density, $\mathrm{FRL}=$ Fresh root length, $\mathrm{RSR}=$ Root - shoot ratio, DRW $=$ Dry root weight, $\mathrm{DSW}=$ Dry shoot weight, $\mathrm{RSWR}=$ Root-shoot weight ratio. 
Table 02: Estimates of genetic components for maize genotypes

\begin{tabular}{lcccccc}
\hline Traits & $\begin{array}{c}\text { Genotypic } \\
\text { Coefficient } \\
\text { Variation } \\
\%\end{array}$ & $\begin{array}{c}\text { Phenotypic } \\
\text { Coefficient } \\
\text { Variation } \\
\%\end{array}$ & $\begin{array}{c}\text { Standard } \\
\text { Deviation }\end{array}$ & $\begin{array}{c}\text { Genotypic } \\
\text { Variance }\end{array}$ & $\begin{array}{c}\text { Phenotypic } \\
\text { Variance }\end{array}$ & $\begin{array}{c}\text { Heritability } \\
\mathrm{h}^{2}{ }_{\mathrm{BS} \%}\end{array}$ \\
\hline $\begin{array}{l}\text { Leaf temperature } \\
\text { Chlorophyll }\end{array}$ & 3.10 & 3.84 & 0.58 & 0.93 & 1.43 & 65.10 \\
$\begin{array}{l}\text { content } \\
\text { Fresh Shoot }\end{array}$ & 130.88 & 131.08 & 0.02 & 0.20 & 0.21 & 99.70 \\
$\begin{array}{l}\text { length } \\
\text { Root density }\end{array}$ & 17.60 & 18.29 & 2.08 & 81.38 & 87.88 & 92.60 \\
$\begin{array}{l}\text { Fresh root length } \\
\text { Root-shoot }\end{array}$ & 14.63 & 16.21 & 0.98 & 18.45 & 18.84 & 83.40 \\
$\begin{array}{l}\text { length ratio } \\
\text { Dry shoot weight }\end{array}$ & 18.92 & 23.94 & 0.05 & 0.01 & 8.89 & 62.40 \\
$\begin{array}{l}\text { Dry root weight } \\
\text { Root-shoot }\end{array}$ & 26.43 & 34.65 & 0.11 & 0.027 & 0.05 & 58.20 \\
weight ratio & 9.65 & 24.00 & 0.16 & 0.01 & 0.05 & 16.20 \\
\hline
\end{tabular}

\section{Correlations}

It is cleared from Table 03 that a positive and significant correlation coefficient of chlorophyll content was found with root density at genotypic and phenotypic levels $(0.91$ \& 0.82 ). Negative and significant correlation coefficient of chlorophyll content was found with leaf temperature both at genotypic and phenotypic levels $(-0.56 \&-0.45)$. Positive and significant association of fresh shoot length was obtained with dry root weight $(0.60 \&$ $0.45)$, dry shoot weight $(0.82 \& 0.55)$, fresh root length (0.58 \& 0.44), leaf temperature $(0.15 \& 0.10)$ and root density $(0.07 \& 0.05)$ both at genotypic and phenotypic levels. Fresh root length indicated a positive and significant correlation coefficient with root shoot length ratio $(0.69 \& 0.55)$, dry root weight $(0.46 \&$ $0.23)$ and fresh shoot length $(0.58 \& 0.44)$ both at genotypic and phenotypic levels. The same results were exhibited in the studies of Ojo et al. (2006). Dry shoot weight was positive and significantly correlated with the root shoot weight ratio $(0.67 \& 0.07)$ and dry root weight $(0.21 \& 0.52)$ at genotypic and phenotypic levels. There was a positive and significant correlation coefficient of dry root weight with fresh root length $(0.46 \& 0.23)$ at genotypic and phenotypic levels. Negative but significant correlation coefficient of dry root weight was found with root density $(-0.15 \&$ $-0.10)$ and leaf temperature $(-0.05 \&-0.44)$ both at genotypic and phenotypic levels. The same results were obtained by Yousuf and Saleem (2001) and Ojo et al. (2006). Positive and significant association occurred between root shoot weight ratio and fresh shoot length $(0.01 \& 0.001)$ only at the phenotypic level. The same results were also obtained in the studies of Ojo et al. (2006) and Malik et al. (2005). The leaf temperature had positive and highly significant association with the root shoot length ratio ( $0.08 \& 0.07)$. RD had negative and significant association with FRL $(-0.08 \&-0.06)$ and LT $(-0.71 \&-0.50)$ both at genotypic and phenotypic levels. 
Table 03: Genotypic and phenotypic correlation for different traits of maize genotypes

\begin{tabular}{|c|c|c|c|c|c|c|c|c|c|}
\hline Variable & $\mathrm{r}$ & Chl.C & DRW & DSW & FRL & LT & $\mathrm{RD}$ & RSLR & RSWR \\
\hline \multirow{2}{*}{ DRW } & G & -0.08 & & & & & & & \\
\hline & $\mathrm{P}$ & -0.06 & & & & & & & \\
\hline \multirow{2}{*}{ DSW } & G & 0.04 & $0.21 * *$ & & & & & & \\
\hline & $\mathrm{P}$ & 0.02 & $0.52 * *$ & & & & & & \\
\hline \multirow{2}{*}{ FRL } & G & 0.04 & $0.46^{*}$ & 0.70 & & & & & \\
\hline & $\mathrm{P}$ & 0.04 & $0.23 * *$ & $0.25^{*}$ & & & & & \\
\hline \multirow[b]{2}{*}{ LT } & G & $-0.56^{* *}$ & $-0.05 * *$ & $-0.18 * *$ & $-0.06 * *$ & & & & \\
\hline & $\mathrm{P}$ & $-0.45^{* *}$ & $-0.44 * *$ & -0.03 & $-0.03 * *$ & & & & \\
\hline \multirow{2}{*}{$\mathrm{RD}$} & G & $0.91 *$ & $-0.15^{* *}$ & $-0.09 * *$ & $-0.08 * *$ & $-0.71 * *$ & & & \\
\hline & $\mathrm{P}$ & $0.82 * *$ & $-0.10 * *$ & 0.02 & $-0.06^{* *}$ & $-0.50^{* *}$ & & & \\
\hline \multirow{2}{*}{ RSLR } & G & -0.09 & -0.15 & -0.11 & $0.69^{* *}$ & $0.08 * *$ & $-0.16^{* *}$ & & \\
\hline & $\mathrm{P}$ & -0.07 & -0.02 & 0.11 & $0.55^{* *}$ & $0.07 * *$ & $-0.08 *$ & & \\
\hline \multirow{2}{*}{ RSWR } & G & -0.17 & $-0.07 * *$ & $0.67 * *$ & $0.06^{* *}$ & $0.13^{*}$ & $-0.16^{*}$ & $0.07 * *$ & \\
\hline & $\mathrm{P}$ & -0.07 & $0.44 * *$ & $0.07^{*}$ & $-0.003 *$ & $-0.05 *$ & $-0.07^{*}$ & $-0.002 *$ & \\
\hline \multirow{2}{*}{ FSL } & G & 0.15 & $0.60^{*}$ & $0.82 *$ & $0.58^{*}$ & $0.15^{*}$ & $0.07^{*}$ & $-0.34 *$ & $0.01 *$ \\
\hline & $\mathrm{P}$ & 0.15 & $0.45^{* *}$ & $0.55^{* *}$ & $0.44 * *$ & $0.10^{* *}$ & $0.05^{*}$ & $-0.31 * *$ & 0.001 \\
\hline
\end{tabular}

$* *=$ Highly significant,$*=$ Significant

DRW $=$ Dry root weight, DSW $=$ Dry shoot weight, FRL $=$ Fresh root length, $\mathrm{LT}=$ Leaf temperature, Chl.C $=$ Chlorophyll contents,

$\mathrm{RD}=$ Root density, $\mathrm{RSLR}=$ Root-shoot length ratio, $\mathrm{RSWR}=$ Root-shoot weight ratio and FSL $=$ Fresh shoot length

Table 04: Direct (In Parenthesis) and indirect effect of various traits on FSL

\begin{tabular}{lcccccccc}
\hline \multicolumn{1}{c}{ Variables } & Chl.C & DRW & DSW & FRL & LT & RD & RSLR & RSWR \\
\hline Chl.C & $(-0.51)$ & -0.01 & 0.02 & 0.03 & -0.19 & 0.66 & 0.06 & 0.09 \\
DRW & 0.04 & $(0.16)$ & 0.65 & 0.34 & -0.02 & -0.11 & 0.10 & -0.57 \\
DSW & -0.02 & 0.20 & $(0.53)$ & 0.52 & -0.06 & -0.07 & 0.08 & -0.36 \\
FRL & -0.02 & 0.08 & 0.37 & $(0.75)$ & -0.02 & -0.06 & -0.48 & -0.03 \\
LT & 0.28 & -0.01 & -0.10 & -0.04 & $(0.35)$ & -0.51 & -0.05 & -0.07 \\
RD & -0.46 & -0.02 & -0.05 & -0.06 & -0.25 & $(0.72)$ & 0.11 & 0.08 \\
RSLR & 0.05 & -0.03 & -0.06 & 0.52 & 0.03 & -0.12 & $(-0.69)$ & -0.04 \\
RSWR & 0.09 & 0.17 & 0.35 & 0.05 & 0.05 & -0.11 & -0.05 & $(-0.54)$ \\
\hline
\end{tabular}

DRW $=$ Dry root weight, $\mathrm{DSW}=$ Dry shoot weight, $\mathrm{FRL}=$ Fresh root length, $\mathrm{LT}=$ Leaf temperature, Chl.C $=$ Chlorophyll content,

$\mathrm{RD}=$ Root density, RSLR $=$ Root-shoot length ratio, RSWR $=$ Root-shoot weight ratio and FSL $=$ Fresh shoot length 


\section{Path coefficient analysis}

It is indicated from Table 04 that the leaf temperature had a positive direct effect on fresh shoot length (0.35) whereas fresh shoot length had negative indirect effects through fresh root length, root density, root shoot length ratio, dry root weight, dry shoot weight and root shoot weight ratio while chlorophyll content had positive indirect effects on fresh shoot length. The direct effect of chlorophyll content on fresh shoot length was negative $(-0.51)$ whereas chlorophyll content had negative indirect effects through leaf temperature and dry root weight while all others had positive indirect effects on fresh shoot length. Similar results were also obtained by Ojo et al. (2006); Rehman et al. (2007) and Ali et al., (2011). The direct effect of root density on fresh shoot length was positive (0.72) whereas root density had negative indirect effects through all traits except root shoot weight ratio and root shoot length ratio which had positive indirect effects on fresh shoot length. The direct effect of fresh root length on fresh shoot length was positive $(0.75)$ whereas fresh root length had negative indirect effects through all traits except dry root weight and dry shoot weight have positive indirect effects on fresh shoot length. The higher direct effects indicated that selection may be useful to be made on the basis of fresh root length for fresh shoot length. The similar results were found by Ojo et al., (2006); Rehman et al., (2007) and Ali et al., (2011). The direct effect of dry root weight on fresh shoot length was positive (0.16) whereas dry root weight had negative indirect effects through all traits except chlorophyll content, dry shoot weight, fresh root length and root shoot length ratio while others had positive indirect effects on fresh shoot length. The direct effects indicated that selection may be made on the basis of dry root weight for fresh shoot length. The direct effect of dry shoot weight on fresh shoot length was positive (0.53) whereas dry shoot weight had negative indirect effects through all traits except dry root weight, fresh root length and root shoot length ratio while others had positive indirect effects through dry shoot weight on fresh shoot length. The similar results were found by O' Regan et al. (1992); Malik et al., (2005) and Ojo et al. (2006). The direct effect of rootshoot weight ratio on fresh shoot length was negative $(-0.54)$ whereas root-shoot weight ratio had positive indirect effects through all traits except root density and root shoot length ratio that had negative indirect effects through dry shoot weight on fresh shoot length. Similar results were obtained by O' Regan et al. (1992); Malik et al. (2005) and Ali et al. (2011). The direct effect of the root shoot length ratio was also negative $(-0.69)$ while the positive indirect effect was due to chlorophyll content, fresh root length and leaf temperature on fresh shoot length.

\section{CONCLUSIONS}

Fresh root length, root density, dry root weight, leaf temperature and dry shoot weight are the traits which contribute largely to the fresh shoot length of maize seedlings. Thus selection could be made for high yielding maize genotypes on the basis of these characters.

\section{REFERENCES}

Ali, Q., M. Elahi, M. Ahsan, M.H.N. Tahir and S.M.A. Basra. (2011). Genetic evaluation of maize (Zea mays L.) genotypes at seedling stage under moisture stress. IJAVMS, 5 (2): 184-193. http://dx.doi.org/10.5455/ijavms.20110608051727

Ali, Q., M. Elahi, M. Ahsan, M.H.N. Tahir and S.M.A. Basra. (2012). Genetic evaluation of maize (Zea mays L.) accessions for growth related seedling traits. IJAVMS, 6 (3): 164172. http://dx.doi.org/10.5455/ijavms.20110608051727 
Anonymous. (2011-12). Economic Survey of Pakistan. Govt. of Pakistan, Finance and Economic Affairs Division, Islamabad, Pakistan: p-21.

Aslam, M. and M. H. N. Tahir. (2003). Correlation and Path Coefficient Analysis of Different Morpho-Physiological Traits of Maize Inbreds under Water Stress Conditions. Inter. J. Agric. \& Bio. 4:446-448.

Chaudhry, A. R. (1983). Maize in Pakistan. Pb. Agri. Res. Coordination Board, Uni. Agri., Faisalabad.

Dewey, D.R. and K.H. Lu. (1959). A correlation and path coefficient analysis of components of crested wheatgrass seed production. Agron. J. 51: 515-518. http://dx.doi.org/10.2134/ agronj1959.00021962005100090002x

Ilker, E. (2011). Correlation and path coefficient analysis in sweet corn. Turk J Field Crops. 16 (2): 105-107.

Kwon, S.H. and J.H. Torrie. (1964). Heritability and interrelationship of two soybean (Glycine $\max$ L.) populations. Crop Sci., 4: 196-198. http://dx.doi.org/10.2135/cropsci1964.00111 83X000400020023x

Malik, H.N., S.I. Malik, M. Hussain, S. U. R. Chughtai and H. I. Javed. (2005). Genetic Correlation among Various Quantitative Characters in Maize (Zea mays L.) Hybrids. J. Agric. \& Social Sci. 3:262-265.

Moulin, S., F. Baret, N. Bruguier and C. Bataille. (2009). Assessing the vertical distribution of leaf chlorophyll content in a maize crop. INRA - Unite Climate, Soil, Environment (CSE), pp: 7803-7929.

O'Ragan, B.P., W.A. Cress and J. Van. Staden. (1992). Response of two maize cultivars to water stress. Pretoria, South Africa, Dept. Agric. 23-25 ISBN 621-4991-8.

Ojo, D.K., O.A. Omikunle, O.A. Oduwaye, M.O. Ajala. (2006). Heritability, Character Correlation and Path Coefficient Analysis among Six Inbred-Lines of Maize (Zea mays L.). World J. Agric. Sci., 2:352-358.

Poethig, R.S., (1988). Heterochronic mutations affecting shoot development in maize. Genetics 119: 959-973.

Rehman, A., S.U. Saleem and G.M. Subhani. (2007). Correlation and path coefficient analysis in Maize (Zea mays l.). J. Agric. Res., 45: 177-183.

Steel, R.G.D., J. H. Torrie and D.A. Dicky.(1997). Principles and Procedures of Statistics. A Biometrical Approach. 3rd Ed. McGraw Hill Book Co. Inc. New York, pp: 400-428.

Thornsberry, J.M., Goodman M.M., Doebley J., Kresovich S., Nielsen D. (2001). Dwarf8 polymorphisms associate with variation in flowering time. Nat. Genet. 28:286289. http://dx.doi.org/10.1038/90135

Yousuf, M. and M. Saleem. (2001). Correlation Analysis of S1 Families of Maize for Grain Yield and its Components. Inter. J. Agric. \& Bio. 4:387-388. 